\title{
A Delay Differential Equation Model of HIV Infection, with Therapy and CTL Response
}

\author{
B. EL Boukari ${ }^{1}$, N. Yousfi ${ }^{2}$ \\ Laboratory Analysis, Modeling and Simulation \\ Department of Mathematics and Computer Science \\ Faculty of Sciences Ben M'sik, University Hassan II, \\ Mohammedia, P.O Box 7955 Sidi Othman, Casablanca,Morocco \\ ${ }^{1}$ Corresponding authors, e-mail: elboukaribrahim@yahoo.fr \\ 2e-mail: nourayousfi@gmail.com
}

Keywords: HIV infection, CTL response, intracellular delay, basic reproduction number, stability.

\begin{abstract}
In this work we investigate a new mathematical model that describes the interactions between CD4+ T cells, human immunodeficiency virus (HIV), immune response and therapy with two drugs. Also an intracellular delay is incorporated into the model to express the lag between the time the virus contacts a target cell and the time the cell becomes actively infected. The model dynamics is completely defined by the basic reproduction number R0. If R0 $\leq 1$ the disease-free equilibrium is globally asymptotically stable, and if R0 $>1$, two endemic steady states exist, and their local stability depends on value of R0. We show that the intracellular delay affects on value of R0 because a larger intracellular delay can reduce the value of R0 to below one. Finally, numerical simulations are presented to illustrate our theoretical results.
\end{abstract}

\section{Introduction}

Mathematical modeling of HIV infection has provided a lot of understandings of the dynamics of HIV infection $[9,12,11,10,4,7]$. In these papers, the authors considered that once a cell is infected, it immediately becomes productively infected and starts producing virus. But, as mentioned in $[3,17]$ there are cascade of events which occur between infection of cell with virus and production of virus at cellular level; this leads to time delay in the infection of the cell.

Culshaw et all in [3] simplify the ODE model proposed by Perelson et al. [10] by considering only three components and they incorporate a discrete delay to the model to describe the time between infection of a CD4+ T-cell and the emission of viral particles on a cellular level. They concluded that, according to specific parameters, the stability for the infected steady state is independent of the delays. But, under another set of assumptions on the parameters, the stability of the steady state depends on the delay and even delay-induced oscillations could occur via instability.

Zhu et all in [17] incorporated a fixed discrete delay into the cell infection equation to model, in order to denotes the lag between the time the virus contacts a target cell and the time the cell becomes actively infected (including the steps of successful attachment of virus to the cell, and penetration of virus into the cell). The model led to the conclusion that large delays can help eliminate the virus.

In [13], the authors proved that the intracellular delays can cause periodic oscillations through Hopf bifurcations. It is also known that Hopf bifurcations can occur in certain classes of HIV models without intracellular delays, in which the target-cell dynamics have a mitosis component given by a logistic term [14].

In this paper, we consider our model presented in [4] which takes account the cure of infected cells, and we incorporate a discrete time delay to the model to describe the time between infection of a CD4+

T-cell and the emission of viral particles on a cellular level as proposed by [17]. In our work, we show that the delay can not cause periodic oscillations.

The paper is organized as follows. The mathematical model is described in section 2. Section 
3 illustrates some properties of the model's solutions. The model analysis and simulation are given in section 4. Discussion, conclusion and future directions of this research are presented in section 5 .

\section{Presentation of model}

We consider the model presented in [4] bay the following system:

$$
\left\{\begin{array}{l}
\dot{x}(t)=\lambda-d x(t)-(1-\eta) \beta v_{I}(t) x(t)+r y(t) \\
\dot{y}(t)=(1-\eta) \beta v_{I}(t) x(t)-(a+r) y(t)-p y(t) z(t) \\
\dot{v}_{I}(t)=(1-\varepsilon) k y(t)-\mu v_{I}(t) \\
\dot{v}_{N I}(t)=\varepsilon k y(t)-\mu v_{N I}(t) \\
\dot{z}(t)=c y(t) z(t)-b z(t)
\end{array}\right.
$$

where the variables are: the concentrations of uninfected CD4+ T-cells (x), infected cells (y), infectious virus (vI ), noninfectious virus (vN I ) and CTLs (z).

Uninfected CD4+ T-cells (x) are produced at a rate $\lambda$, die at a rate $\mathrm{d}$ and become infected by virus at a rate $\beta$. Infected cells (y) die at a rate a. Free virus ( $\mathrm{vN} \mathrm{I}$ and $\mathrm{vI})$ are produced by infected cells at a rate $\mathrm{k}$ and cleared at a rate $\mu . \mathrm{p}$ is the infected cells elimination rate by the CTL response, $\mathrm{c}$ is the CTL reproduction rate and $\mathrm{b}$ is the CTL death rate. In addition, through therapy, a part of infected cells may also revert to the uninfected state by loss of all cccDNA from their nucleus at a rate $r$. The parameters $\varepsilon$ and $\eta$ measure the efficacy of reverse transcriptase inhibitor (RTIs) and protease inhibitor (PIs) respectively.

In the model (1), we introduce a time delay between infection of a cell and production of new virus particles, we assume that virus production lags by a delay $\tau$ behind the infection of a cell. This implies that recruitment of virus producing cells at time $t$ is not given by the density $(1-\eta) \beta \mathrm{vI}$ $(t) x(t)$ of newly infected cells, but rather by the density of cells that were newly infected at time $t-$ $\tau$ and are still alive at time t. We assume that the death rate a for infected but not yet virusproducing cells, Therefore, the - a $\tau$ probability of surviving from time $t-\tau$ to time $t$ is $e^{-a T}$. Thus, we obtain the following model:

$$
\left\{\begin{array}{l}
\dot{x}(t)=\lambda-d x(t)-(1-\eta) \beta v_{I}(t) x(t)+r y(t) \\
\dot{y}(t)=(1-\eta) \beta e^{-a \tau} v_{I}(t-\tau) x(t-\tau)-(a+r) y(t)-p y(t) z(t) \\
\dot{v}_{I}(t)=(1-\varepsilon) k y(t)-\mu v_{I}(t) \\
\dot{v}_{N I}(t)=\varepsilon k y(t)-\mu v_{N I}(t) \\
\dot{z}(t)=c y(t) z(t)-b z(t)
\end{array}\right.
$$

We put $\theta=\varepsilon+\eta-\varepsilon \eta$, wich represents the combined efficacy of the two drugs. Then $1-\theta=(1-$ $\varepsilon)(1-\eta)$ which implies that each drug acts independently.

We define the basic reproduction number R0 of the model (2) by

$$
R_{0}=\frac{(1-\theta) \beta k \lambda e^{-a \tau}}{d \mu(a+r)}
$$

Recall that R0 is defined as the average number of secondary infections produced by one infected cell over its average life time 1 when all cells are uninfected.

\subsection{Parameters estimation}

Ciupe et all in [2], based on Monte Carlo method, estimated the death rate of infected cells, a, to be between 0.00019 day -1 and 0.711 day -1 and the total number of viral particles produced by an 
infected cell during its lifetime, $\mathrm{N}$, was estimated by [2] to be between 160.26 and 591851 . Hence the production rate of virus by infected cells, $\mathrm{k}$, is between 0.0304 day -1 and 420806.061 day -1 , because we have the relation $\mathrm{k}=\mathrm{aN}$.

The other parameters are given in the following table.

Table 1: Parameters, their symbols and default values used in the model (2)

\begin{tabular}{|c|l|l|}
\hline Parameters & Value & References \\
\hline$\lambda$ & $5-10$ day $^{-1} \mathrm{~mm}^{-3}$ & {$[1,3,7,8,10,13,16,17]$} \\
\hline $\mathrm{d}$ & $0.01-0.03 \mathrm{day}^{-1}$ & {$[1,2,3,7,8,10,13,16,17]$} \\
\hline$\beta$ & $\begin{array}{l}24.10^{-6}-48.10^{-4} \\
\mathrm{~mm}^{3} \mathrm{day}^{-1}\end{array}$ & {$[1,2,3,7,8,10,13,16,17]$} \\
\hline$\eta$ & $0-1$ & {$[7]$} \\
\hline$\varepsilon$ & $0-1$ & {$[7]$} \\
\hline $\mathrm{r}$ & 0.01 day $^{-1}$ & {$[7]$} \\
\hline $\mathrm{a}$ & $0.00019-0.711$ day $^{-1}$ & {$[1,2,3,7,8,10,13,16,17]$} \\
\hline $\mathrm{k}$ & $0.0304-420806.061$ & Calculated \\
\hline$\mu$ & day & {$[1,3,7,8,10,13,16,17]$} \\
\hline $\mathrm{c}$ & $1.8-3$ day $^{-1}$ & {$[17]$} \\
\hline $\mathrm{b}$ & 0.2 day $^{-1} \mathrm{~mm}^{3}$ & {$[1,13,17]$} \\
\hline $\mathrm{p}$ & $0.1-0.3$ day $^{-1}$ & {$[1,13,17]$} \\
\hline & $0.01-0.05$ day $^{-1} \mathrm{~mm}^{3}$ & {[} \\
\hline
\end{tabular}

\section{Properties of solutions}

\subsection{Positivity and boundedness of solutions}

In this section, we establish the positivity and boundedness of solutions of model (2) Since this model describes the evolution of a cell population, the cell densities must remain non-negative and bounded. These properties imply global existence of the solutions.

Let $\mathrm{C}=\mathrm{C}([-\tau, 0], \mathrm{R} 5)$ be the Banach space of continuous functions mapping the interval $[-\tau, 0]$ into R5 with the topology of uniform convergence. By the fundamental theory of functional differential equations (see J. Hale et all [6]), it is easy to show that there exists a unique solution $(\mathrm{x}(\mathrm{t}), \mathrm{y}(\mathrm{t}), \mathrm{vI}(\mathrm{t}), \mathrm{vN} \mathrm{I}(\mathrm{t}), \mathrm{z}(\mathrm{t}))$ of system (2) with initial data $(\mathrm{x} 0, \mathrm{y} 0, \mathrm{vI} 0, \mathrm{vN} \mathrm{I} 0, \mathrm{z} 0) \in \mathrm{C}$.

In addition, for biological reasons, we assume that the initial data for system (2) satisfy:

$$
x 0(s) \geq 0, y 0(s) \geq 0, v I 0(s) \geq 0, v N I 0(s) \geq 0, z 0(s) \geq 0, \text { for all } s \in[-\tau, 0]
$$

\section{Proposition 1}

All solutions of system (2) subject to condition (3) remain bounded and non-negative for all $t \in[0,+\infty[$.

Moreover, we have
i) $T(t) \leq T(0)+\frac{\lambda}{\delta}$
ii) $v_{I}(t) \leq v_{I}(0)+\frac{(1-\varepsilon) k}{\mu}\|y\|_{\infty}$
iii) $v_{N I}(t) \leq v_{N I}(0)+\frac{\varepsilon k}{\mu}\|y\|_{\infty}$

where $T(t)=x(t)+y(t)+\frac{p}{c} z(t)+(1-\eta) \beta \int_{t-\tau}^{t} e^{-a(t-s)} x(s) v_{I}(s) d s$ and $\delta=\min (a, b, d)$ 


\section{Proof.}

From system (2) we have:

$$
\begin{gathered}
x(t)=e^{-d t}\left[x(0) e^{-(1-\eta) \beta \int_{0}^{t} v_{I}(u) d u}+\int_{0}^{t}(\lambda \mid+r y(s)) e^{d s-(1-\eta) \beta \int_{s}^{t} v_{I}(u) d u} d s\right] \\
y(t)=y(0) e^{-\int_{0}^{t}(a+r+p z(s)) d s}+\int_{0}^{t}(1-\eta) \beta v_{I}(w-\tau) x(w-\tau) e^{-a \tau} e^{-\int_{w}^{t}(a+r+p z(s)) d s} d w \\
v_{I}(t)=v_{I}(0) e^{-\mu t}+(1-\varepsilon) k \int_{0}^{t} y(s) e^{\mu(s-t)} d s \\
v_{N I}(t)=v_{N I}(0) e^{-\mu t}+\varepsilon k \int_{0}^{t} y(s) e^{\mu(s-t)} d s \\
z(t)=z(0) e^{-\int_{0}^{t}(b-c y(s)) d s}
\end{gathered}
$$

Let $\mathrm{t} \in[0, \tau]$, we have $\mathrm{s}-\tau \in[-\tau, 0]$ for all $\mathrm{s} \in[0, \tau]$. Using (3) and (5), we deduce that $\mathrm{y}(\mathrm{t}) \geq 0$ for $t \in[0, \tau]$. Consequently we have that $x(t), v I(t), v N ~ I(t)$, and $z(t)$ are all non negative on the interval $[0, \tau]$. This method can now be repeated to deduce non-negativity of $\mathrm{x}, \mathrm{y}, \mathrm{vI}, \mathrm{vN} \mathrm{I}$, and $\mathrm{z}$ on the interval $[\tau, 2 \tau]$ and then on successive intervals $[n \tau,(n+1) \tau], n \geq 2$, to include all positive times. This proves the positivity of solutions.

As $\dot{T}(t)=\lambda-d x(t)-a y(t)-\frac{p b}{c} z(t)-a(1-\eta) \beta \int_{t-\tau}^{t} e^{-a(t-s)} x(s) v_{I}(s) d s$.

we deduce that $\dot{T}(t) \leq \lambda-\delta T(t)$. ie $T(t) \leq T(0) e^{-\delta t}+\frac{\lambda}{\delta}\left(1-e^{-\delta t}\right)$.

Since $0 \leq e^{-\delta t} \leq 1$ and $1-e^{-\delta t} \leq 1$ thus $\left.i\right)$ Now we show ii), From relation (6) we deduce $v_{I}(t) \leq$ $v_{I}(0) e^{-\mu t}+\frac{(1-\varepsilon) k}{\mu}\|y\|_{\infty}\left(1-e^{-t \mu}\right)$.

Since $1-e^{-t \mu} \leq 1$, and $e^{-t \mu} \leq 1$ we deduce ii).

By the same technique that ii), we show iii).

\subsection{Non-periodicity and Limiting Behavior}

\section{Proposition 2}

Let $\mathrm{V}(\mathrm{t})=(\mathrm{x}(\mathrm{t}), \mathrm{y}(\mathrm{t}), \mathrm{vI}(\mathrm{t}), \mathrm{vN} \mathrm{I}(\mathrm{t}), \mathrm{z}(\mathrm{t}))$ be a solution of system (2) subject to condition (3), then the limit of $V(t)$ exists when $t \rightarrow+\infty$.

In particular, $\mathrm{V}$ is periodic if and only if $\mathrm{V}$ is stationary. Moreover, we have:

$$
\begin{aligned}
\lim _{t \rightarrow+\infty} x(t) & \leq \frac{\lambda}{d} \\
\lim _{t \rightarrow+\infty} y(t) & \leq \frac{\lambda\left(\lambda-d \lim _{t \rightarrow+\infty} x(t)\right)}{a \lambda+d R_{0}(a+r)\left(e^{a \tau}-1\right) \lim _{t \rightarrow+\infty} x(t)} \\
\lim _{t \rightarrow+\infty} v_{I}(t) & =\frac{(1-\epsilon) k}{\mu} \lim _{t \rightarrow+\infty} y(t) \\
\lim _{t \rightarrow+\infty} v_{N I}(t) & =\frac{\epsilon k}{\mu} \lim _{t \rightarrow+\infty} y(t) \\
\lim _{t \rightarrow+\infty} z(t) & =\frac{c}{p b}\left(\lambda-d \lim _{t \rightarrow+\infty} x(t)-a \lim _{t \rightarrow+\infty} y(t)-R_{0} d(a+r)\left(e^{a \tau}-1\right) \lim _{t \rightarrow+\infty} x(t) \lim _{t \rightarrow+\infty} y(t)\right)
\end{aligned}
$$


From system(2) we have

$$
\dot{y}+a y=\lambda-(\dot{x}+d x)-\frac{p}{c}(\dot{z}+b z)-(\dot{l}+a l)
$$

with $l(t)=(1-\eta) \beta \int_{t-\tau}^{t} e^{-a(t-s)} x(s) v_{I}(s) d s$. Hence

$$
\left(y e^{a t}\right)^{\prime}=\lambda e^{a t}-\left(x e^{d t}\right)^{\prime} e^{(a-d) t}-\frac{p}{c}\left(z e^{b t}\right)^{\prime} e^{(a-b) t}-\left(l e^{a t}\right)^{\prime}
$$

Using the integration by parts,we get

$$
\begin{aligned}
& \int_{0}^{t}\left(x(s) e^{d s}\right)^{\prime} e^{(a-d) s} d s=x(t) e^{a t}-x(0)-(a-d) \int_{0}^{t} x(s) e^{a s} d s \\
& \int_{0}^{t}\left(z(s) e^{b s}\right)^{\prime} e^{(a-b) s} d s=z(t) e^{a t}-z(0)-(a-b) \int_{0}^{t} z(s) e^{a s} d s
\end{aligned}
$$

hence

$$
\begin{aligned}
y(t)=\left(x_{0}+y_{0}+\frac{p}{c} z_{0}\right. & +l(0)) e^{-a t}+\frac{\lambda}{a}\left(1-e^{-a t}\right)-\left(x(t)+\frac{p}{c} z(t)+l(t)\right) \\
& +\int_{0}^{t}\left[(a-d) x(s)+\frac{p}{c}(a-b) z(s)\right] e^{a(s-t)} d s
\end{aligned}
$$

If $\max (a, b, d)=a$, according to lemma 3.3 (see Yousfi et all [15]), we have

$$
\begin{aligned}
& \limsup _{t \rightarrow+\infty} y(t) \leq \frac{1}{a}\left[\lambda-d \limsup _{t \rightarrow+\infty} x(t)-\frac{p b}{c} \limsup _{t \rightarrow+\infty} z(t)-a \limsup _{t \rightarrow+\infty} l(t)\right] \\
& \liminf _{t \rightarrow+\infty} y(t) \geq \frac{1}{a}\left[\lambda-d \liminf _{t \rightarrow+\infty} x(t)-\frac{p b}{c} \liminf _{t \rightarrow+\infty} z(t)-a \liminf _{t \rightarrow+\infty} l(t)\right]
\end{aligned}
$$

If $\max (a, b, d)=b$, from (9), and using the same technique as in (10), we have

$$
\begin{aligned}
z(t)=\frac{c}{p}[(x(0) & \left.+y(0)+\frac{p}{c} z(0)+l(0)\right) e^{-b t}+\frac{\lambda}{b}\left(1-e^{-b t}\right)-(x(t)+y(t)+l(t)) \\
& \left.+\int_{0}^{t}((b-d) x(s)+(b-a) y(s)+(b-a) l(s)) e^{b(s-t)} d s\right] .
\end{aligned}
$$

According to lemma 3.3 ([15]), we have

$$
\begin{aligned}
\limsup _{t \rightarrow \perp \sim} z(t) & \leq \frac{c}{n h}\left[\lambda-d \limsup _{t \rightarrow \pm \sim} x(t)-a \limsup _{t \rightarrow \perp \sim} y(t)-a \limsup _{t \rightarrow \pm \sim} l(t)\right] \\
\liminf _{t \rightarrow+\infty} z(t) & \geq \frac{c}{p b}\left[\lambda-d \liminf _{t \rightarrow+\infty} x(t)-a \liminf _{t \rightarrow+\infty} y(t)-a \liminf _{t \rightarrow+\infty} l(t)\right]
\end{aligned}
$$

If $\max (a, b, d)=d$, from (9), and using the same technique as in (10), we have

$$
\begin{aligned}
x(t)=(x(0)+y(0) & \left.+\frac{p}{c} z(0)+l(0)\right) e^{-d t}+\frac{\lambda}{d}\left(1-e^{-d t}\right)-\left(y(t)+\frac{p}{c} z(t)+l(t)\right) \\
& +\int_{0}^{t}\left[(d-a) y(s)+\frac{p}{c}(d-b) z(s)+(d-a) l(s)\right] e^{d(s-t)} d s
\end{aligned}
$$

According to lemma 3.3 ([15]), we have

$$
\begin{aligned}
& \limsup _{t \rightarrow+\infty} x(t) \leq \frac{1}{d}\left[\lambda-a \limsup _{t \rightarrow+\infty} y(t)-\frac{p b}{c} \limsup _{t \rightarrow+\infty} z(t)-a \limsup _{t \rightarrow+\infty} l(t)\right] \\
& \liminf _{t \rightarrow+\infty} x(t) \geq \frac{1}{d}\left[\lambda-a \liminf _{t \rightarrow+\infty} y(t)-\frac{p b}{c} \liminf _{t \rightarrow+\infty} z(t)-a \liminf _{t \rightarrow+\infty} l(t)\right]
\end{aligned}
$$


So, for every parameters value $\mathrm{a}>0, \mathrm{~b}>0$ and $\mathrm{d}>0$, we have

$$
\begin{aligned}
& \limsup _{t \rightarrow+\infty} z(t) \leq \frac{c}{p b}\left[\lambda-d \limsup _{t \rightarrow+\infty} x(t)-a \limsup _{t \rightarrow+\infty} y(t)-a \limsup _{t \rightarrow+\infty} l(t)\right] \\
& \liminf _{t \rightarrow+\infty} z(t) \geq \frac{c}{p b}\left[\lambda-d \liminf _{t \rightarrow+\infty} x(t)-a \liminf _{t \rightarrow+\infty} y(t)-a \liminf _{t \rightarrow+\infty} l(t)\right]
\end{aligned}
$$

Hence,

$$
\begin{aligned}
\limsup _{t \rightarrow+\infty} z(t)-\liminf _{t \rightarrow+\infty} z(t) \leq \frac{c}{p b}\left[d\left(\liminf _{t \rightarrow+\infty} x(t)-\limsup _{t \rightarrow+\infty} x(t)\right)\right. & +a\left(\liminf _{t \rightarrow+\infty} y(t)-\limsup _{t \rightarrow+\infty} y(t)\right) \\
& \left.+a\left(\liminf _{t \rightarrow+\infty} l(t)-\limsup _{t \rightarrow+\infty} l(t)\right)\right]
\end{aligned}
$$

Thus,

$$
\begin{aligned}
& \limsup _{t \rightarrow+\infty} x(t)=\liminf _{t \rightarrow+\infty} x(t) \\
& \limsup _{t \rightarrow+\infty} y(t)=\liminf _{t \rightarrow+\infty} y(t) \\
& \limsup _{t \rightarrow+\infty} z(t)=\liminf _{t \rightarrow+\infty} z(t)
\end{aligned}
$$

Hence

$$
\begin{aligned}
& \lim _{t \rightarrow+\infty} x(t)=\frac{1}{d}\left[\lambda-a \lim _{t \rightarrow+\infty} y(t)-\frac{p b}{c} \lim _{t \rightarrow+\infty} z(t)-a \lim _{t \rightarrow+\infty} l(t)\right] \\
& \lim _{t \rightarrow+\infty} z(t)=\frac{c}{p b}\left(\lambda-d \lim _{t \rightarrow+\infty} x(t)-a \lim _{t \rightarrow+\infty} y(t)-a \lim _{t \rightarrow+\infty} l(t)\right)
\end{aligned}
$$

As $y(t)>0, z(t)>0$, and $1(t)>0$ for all $t>0,(13)$ implies that

$$
\lim _{t \rightarrow+\infty} x(t) \leq \frac{\lambda}{d}
$$

From (6), and using lemma 3.3 ([15]) we have

Also from (7), and lemm:

$$
\lim _{t \rightarrow+\infty} v_{I}(t)=\frac{(1-\epsilon) k}{\mu} \lim _{t \rightarrow+\infty} y(t) .
$$

$$
\lim _{t \rightarrow+\infty} v_{N I}(t)=\frac{\epsilon k}{\mu} \lim _{t \rightarrow+\infty} y(t) .
$$

From system (2) we have

$$
\dot{y}+a y \leq \lambda-(\dot{x}+d x)-(1-\eta) \beta\left[-e^{-a \tau} v_{I}(t-\tau) x(t-\tau)+v_{I}(t) x(t)\right] .
$$

Implies that

$$
\dot{y}+a y \leq \lambda-(\dot{x}+d x)-(\dot{l}+a l) .
$$

Hence

$$
y(t) \leq(x(0)+y(0)+l(0)) e^{-a t}+\frac{\lambda}{a}\left(1-e^{-a t}\right)-(x(t)+l(t))+\int_{0}^{t}(a-d) x(s) e^{a(s-t)} d s .
$$

Then

$$
\lim _{t \rightarrow+\infty} y(t) \leq \frac{\lambda}{a}-\frac{d}{a} \lim _{t \rightarrow+\infty} x(t)-\lim _{t \rightarrow+\infty} l(t) .
$$


We have

$$
l(t)=(1-\eta) \beta\left[\int_{0}^{t} e^{-a(t-s)} x(s) v_{I}(s) d s-\int_{0}^{t-\tau} e^{-a(t-s)} x(s) v_{I}(s) d s .\right.
$$

Using lemma 3.3 ([15]) and (6) we have

$$
\lim _{t \rightarrow+\infty} l(t)=\frac{(1-\eta) \beta}{a}\left(1-e^{-a \tau}\right) \lim _{t \rightarrow+\infty} x(t) \lim _{t \rightarrow+\infty} v_{I}(t) .
$$

This implies

$$
\lim _{t \rightarrow+\infty} l(t)=\frac{(1-\eta) \beta}{a} \frac{(1-\varepsilon) k}{\mu}\left(1-e^{-a \tau}\right) \lim _{t \rightarrow+\infty} x(t) \lim _{t \rightarrow+\infty} y(t) .
$$

From (15) and (16) we deduce

$$
\lim _{t \rightarrow+\infty} y(t) \leq \frac{\lambda\left(\lambda-d \lim _{t \rightarrow+\infty} x(t)\right)}{a \lambda+d R_{0}(a+r)\left(e^{a \tau}-1\right) \lim _{t \rightarrow+\infty} x(t)} .
$$

From (14) and (16) we deduce

$$
\lim _{t \rightarrow+\infty} z(t)=\frac{c}{p b}\left(\lambda-d \lim _{t \rightarrow+\infty} x(t)-a \lim _{t \rightarrow+\infty} y(t)-R_{0} d(a+r)\left(e^{a \tau}-1\right) \lim _{t \rightarrow+\infty} x(t) \lim _{t \rightarrow+\infty} y(t)\right)
$$

\section{Analysis of the model}

In this section we show that there exist three equilibrium points: disease free equilibrium point, endemic equilibrium without immune response, and endemic equilibrium with immune response. We will study the stability of these equilibrium points.

\subsection{Equilibrium points}

The system (2) always has a disease free equilibrium $\mathrm{E}_{\mathrm{f}}=((\lambda l d), 0,0,0,0)$ level of healthy $\mathrm{CD} 4^{+}$ T-cells, and two endemic equilibrium points:

$$
\begin{gathered}
\mathrm{E}_{1}=\left(\frac{\lambda}{d R_{0}}, \frac{\lambda\left(R_{0}-1\right) e^{-a \tau}}{a_{r \tau} R_{0}}, \frac{(a+r) d\left(R_{0}-1\right)}{a_{r \tau} \beta(1-\eta)}, \frac{(a+r) \epsilon d\left(R_{0}-1\right)}{a_{r \tau} \beta(1-\theta)}, 0\right) \\
\mathrm{E}_{2}=\left(\frac{\lambda}{d}\left(\frac{r b+\lambda c}{c \lambda+R_{0} b(a+r) e^{a \tau}}\right), \frac{b}{c}, \frac{(1-\epsilon) k b}{\mu c}, \frac{\epsilon k b}{\mu c}, \frac{(a+r)}{p}\left(\frac{-c \lambda+R_{0}(c \lambda+r b)-R_{0}(a+r) b e^{a \tau}}{c \lambda+R_{0} b(a+r) e^{a \tau}}\right)\right)
\end{gathered}
$$

where ar $\tau=\mathrm{a}_{\mathrm{rr}}+\mathrm{r}-\mathrm{re}^{-a T}$

From the biological point of view, the point E1 represents absence of CTL immune response and E2 represents HIV chronic state of disease with CTL response.

\subsection{Stability of free equilibria}

Let $\mathrm{E}=(\mathrm{x}, \mathrm{y}, \mathrm{vI}, \mathrm{vN} \mathrm{I}, \mathrm{z})$ bet any arbitrary equilibrium. Then the characteristic equation about $\mathrm{E}$ is given by:

$$
\left|\begin{array}{ccccc}
-d-(1-\eta) \beta v_{I}-\xi & r & -(1-\eta) \beta x & 0 & 0 \\
(1-\eta) \beta v_{I} e^{-(a+\xi) \tau} & -(a+r)-p z-\xi & (1-\eta) \beta x e^{-(a+\xi) \tau} & 0 & -p y \\
0 & (1-\epsilon) k & -\mu-\xi & 0 & 0 \\
0 & \epsilon k & 0 & -\mu-\xi & 0 \\
0 & c z & 0 & 0 & c y-b-\xi
\end{array}\right|=0
$$

Here we study the stability of the equilibrium point $\mathrm{Ef}=((\lambda \backslash d), 0,0,0,0)$ 


\section{Theorem 1}

i) If $R_{0} \leq 1$, then the disease free equilibrium, $E_{f}$, is globally asymptotically stable.

ii) If $R_{0}>1$, then $E_{f}$ is unstable.

\section{Proof.}

We first show i), using proposition 2 , we have limt $\rightarrow+\infty X(t)$ is finite.

According to Barbalat's lemma, we have: $\lim _{t \rightarrow+\infty} X^{*}(t)=(0,0,0,0,0)$. Hence $\lim _{t \rightarrow+\infty} X(t)=E_{f}$ or $\lim _{\mathrm{t} \rightarrow+\infty} X(t)=\mathrm{E}_{1}$ or $\lim _{\mathrm{t} \rightarrow+\infty} X(t)=\mathrm{E}_{2}$

- If $R_{0}<1$ then $\mathrm{E}_{1}$ and $\mathrm{E}_{2}$ do not exist Hence $\lim _{t \rightarrow+\infty} \mathrm{X}(t)=\mathrm{E}_{f}$.

- If $R_{0}=1$ then $\mathrm{E}_{1}=\mathrm{E}_{f}$ and $\mathrm{E}_{2}$ do not exist Hence $\lim _{\mathrm{t} \rightarrow+\infty} \mathrm{X}_{(t)}=\mathrm{E}_{f}$.

To show ii), we use the Jacobian matrix of Ef The characteristic equation corresponding to $\mathrm{E}_{f}$ is given by:

$$
\left|\begin{array}{ccccc}
-d-\xi & r & -(1-\eta) \beta \frac{\lambda}{d} & 0 & 0 \\
0 & -(a+r)-\xi & (1-\eta) \beta \frac{\lambda}{d} e^{-(a+\xi) \tau} & 0 & 0 \\
0 & (1-\epsilon) k & -\mu-\xi & 0 & 0 \\
0 & \epsilon k & 0 & -\mu-\xi & 0 \\
0 & 0 & 0 & 0 & -b-\xi
\end{array}\right|=0
$$

(18) reduces to

$(\mathrm{d}+\xi)(\mu+\xi)(\mathrm{b}+\xi)[\xi 2+(\mathrm{a}+\mathrm{r}+\mu) \xi+\mu(\mathrm{a}+\mathrm{r})(1-\mathrm{R} 0 \mathrm{e}-\tau \xi)]=0$

The roots $\xi 1=-\mathrm{d}, \xi 2=-\mu, \xi 3=-\mathrm{b}$ are negative. On the other hand, it easy to show that the function, $\mathrm{f}(\xi)=\xi 2+(\mathrm{a}+\mathrm{r}+\mu) \xi+\mu(\mathrm{a}+-\tau \xi \mathrm{r})(1-\mathrm{R} 0 \mathrm{e})$, has a real positive root when $\mathrm{R} 0>1$. Because we have $\mathrm{f}(0)=(\mathrm{a}+\mathrm{r}) \mu(1-\mathrm{R} 0)<0$, if $\mathrm{R} 0>1$ and $\lim \xi \rightarrow+\infty \mathrm{f}(\xi)=+\infty$, and $\mathrm{f}$ is increasing and continuous on $[0,+\infty[$. Consequently, $f$ has a positive real root and the disease free equilibrium $\mathrm{Ef}$ is unstable if $\mathrm{R} 0>1$.
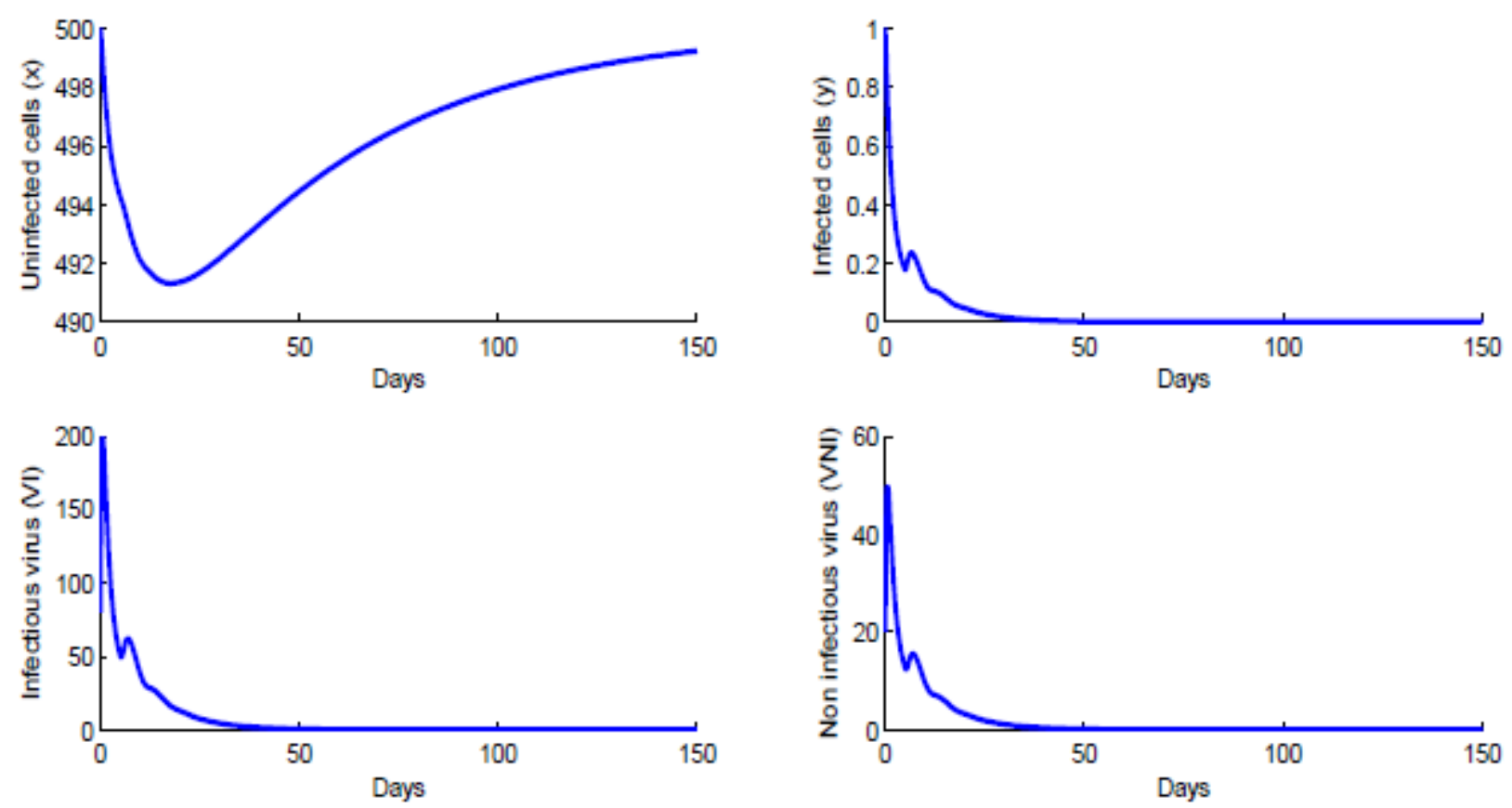


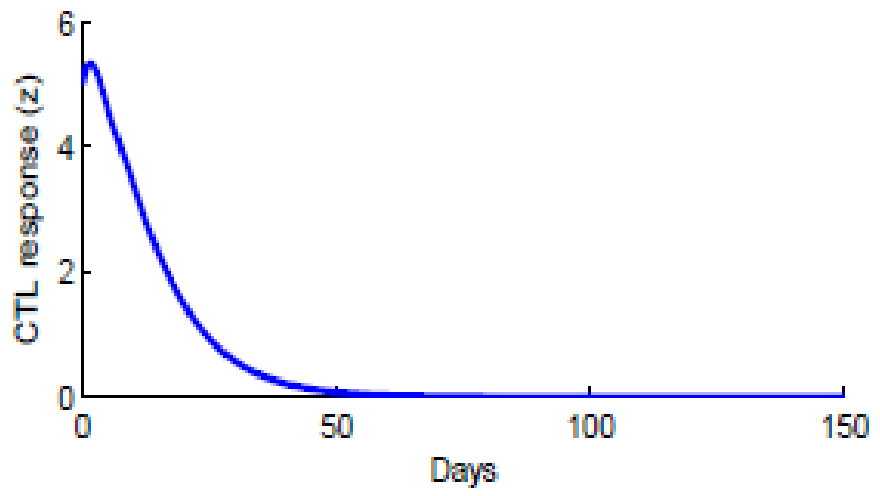

Figure 1: Shows the stability of $E_{\mathrm{f}}$. The parameters are $\varepsilon=0.2, \eta=0.1, \lambda=10, \beta=0.000024$, $\mathrm{k}=1000, \mathrm{a}=0.5, \mathrm{r}=0.01, \mathrm{~d}=0.02, \mu=3, \mathrm{c}=0.2, \mathrm{~b}=0.1, \mathrm{p}=0.01 . \tau=5$ In this case, $\mathrm{R}_{0}=0.4635$ and $\mathrm{E}_{\mathrm{f}}(500,0,0,0,0)$.

\subsection{Stability of the equilibrium without immune response}

Here we study the existence and stability of the endemic equilibrium point without immune

response, $\mathrm{E}_{1}$.

\section{Theorem 2}

i) If $R_{0}<1$, then the point $E_{1}$ does not exist; and $E_{1}=E_{f}$ when $R_{0}=1$.

ii) If $R_{0}>1$, and $R_{1}<1$, then $E_{1}$ is locally asymptotically stable.

iii) If $R_{1}>1$, then $E_{1}$ is unstable.

\section{Proof.}

It easy to verify that if $R_{0}<1$, then the point $E_{1}$ does not exists and $E_{1}=E_{f}$ when $R_{0}=1$.

From (17), the characteristic equation corresponding to E1 reduces to

$\left[\frac{c \lambda+b(a+r) R_{0} e^{a \tau}}{a_{r \tau} R_{0} e^{a \tau}}\left(R_{1}-1\right)-\xi\right][\mu+\xi]\left[\xi^{3}+a_{1}(\tau) \xi^{2}+a_{2}(\tau) \xi+a_{3}(\tau)+\left(b_{1}(\tau)+b_{2}(\tau) \xi\right) e^{-\xi \tau}\right]=0(19)$

where

$$
\begin{aligned}
& a_{1}(\tau)=a+r+\mu+d+\frac{(a+r) d\left(R_{0}-1\right)}{a_{r \tau}} \\
& a_{2}(\tau)=(a+r) \mu+(a+r+\mu)\left[d+\frac{(a+r) d\left(R_{0}-1\right)}{a_{r \tau}}\right] \\
& a_{3}(\tau)=(a+r) \mu\left[d+\frac{(a+r) d\left(R_{0}-1\right)}{a_{r \tau}}\right] \\
& b_{1}(\tau)=-(a+r) d \mu\left[\frac{r\left(R_{0}-1\right) e^{-a \tau}}{a_{r \tau}}+1\right] \\
& b_{2}(\tau)=-(a+r)\left[\frac{r d\left(R_{0}-1\right) e^{-a \tau}}{a_{r \tau}}+\mu\right]
\end{aligned}
$$

We have: the eigenvalue $\xi 1=-\mu$ of (19) is no positive, and the eigenvalue

$\xi_{2}=\frac{c \lambda+b(a+r) R_{0} e^{a \tau}}{a_{r \tau} R_{0} e^{a \tau}}\left(R_{1}-1\right)$ s no positive if $R_{1}<1$. Then the stability of $E_{1}$ is determined by the distribution of the roots of equation

$$
\xi^{3}+a_{1}(\tau) \xi^{2}+a_{2}(\tau) \xi+a_{3}(\tau)+\left(b_{1}(\tau)+b_{2}(\tau) \xi\right) e^{-\xi \tau}=0
$$


If $\tau=0$, equation (20) reduces to

$$
\xi^{3}+c_{1} \xi^{2}+c_{2} \xi+c_{3}=0
$$

where

$$
\begin{aligned}
& c_{1}=a+r+\mu+d R_{0}+\frac{r d\left(R_{0}-1\right)}{a} \\
& c_{2}=(a+r+\mu) d R_{0}+\frac{\mu r d\left(R_{0}-1\right)}{a} \\
& c_{3}=(a+r) d \mu\left(R_{0}-1\right)
\end{aligned}
$$

Using the same technique as that defined in [7] by K.Hattaf and N.Yousfi, and from the RouthHurwitz theorem given in [5], all the roots of equation (21) have negative real parts if $R_{0}>1$.

If $\tau>0$, equation (20) has infinitely many roots. By Rouche's Theorem and continuity in $\tau$, the sign of the roots of equation (20) will change if it crosses imaginary axis, i.e. if Eqs (20) has purely imaginary. Let $\xi=i \omega$ with $\omega>0$ be a purely imaginary root of (20). Then,

$-\omega^{3} i-a_{1}(\tau) \omega^{2}+a_{2}(\tau) i \omega+a_{3}(\tau)=-\left(b_{1}(\tau)+b_{2}(\tau) i \omega\right) e^{-i \omega \tau}$

Taking the modulus in the above equation and grouping in terms of the powers of $\omega$, we obtain the following equation

$$
\omega^{6}+\left[a_{1}^{2}(\tau)-2 a_{2}(\tau)\right] \omega^{4}+\left[a_{2}^{2}(\tau)-2 a_{1}(\tau) a_{3}(\tau)-b_{2}^{2}(\tau)\right] \omega^{2}+a_{3}^{2}(\tau)-b_{1}^{2}(\tau)=0
$$

where

$$
\begin{aligned}
a_{1}^{2}(\tau)-2 a_{2}(\tau) & =(a+r)^{2}+\mu^{2}+d^{2}\left(1+\frac{(a+r)\left(R_{0}-1\right)}{a_{r \tau}}\right)^{2}>0 \\
a_{2}^{2}(\tau)-2 a_{1}(\tau) a_{3}(\tau)-b_{2}^{2}(\tau) & =\frac{(a+r)^{2} d}{a_{r \tau}^{2}}\left[2 a_{r \tau} r d \mu\left(R_{0}-1\right) e^{-a \tau}+\left(a^{2}+\mu^{2}+2 a r\right) R_{0}^{2}\right. \\
& \left.+r^{2}\left[R_{0}+\left(R_{0}-1\right) e^{-a \tau}\right]\left[R_{0}\left(1-e^{-a \tau}\right)+e^{-a \tau}\right]\right]>0 \\
a_{3}^{2}(\tau)-b_{1}^{2}(\tau) & =\left(a_{3}(\tau)-b_{1}(\tau)\right)(a+r) d \mu\left(R_{0}-1\right)>0
\end{aligned}
$$

Let $X=\omega 2$ then $(22)$ becomes

$$
F(X)=X^{3}+\left[a_{1}^{2}(\tau)-2 a_{2}(\tau)\right] X^{2}+\left[a_{2}^{2}(\tau)-2 a_{1}(\tau) a_{3}(\tau)-b_{1}^{2}(\tau)\right] X+a_{3}^{2}(\tau)-b_{1}^{2}(\tau)=0 .
$$

The function $\mathrm{F}$ is increasing in $\mathrm{X} \in[0,+\infty[$ with

$F(0)=(a+r)^{2} d^{2} \mu^{2}\left(R_{0}-1\right)\left[2+\left(\frac{R_{0}-1}{a_{r \tau}}\right)\left(a+r+r e^{-a T}\right)\right]>0$.

Then (23) has no positive solutions if $\mathrm{R}_{0}>1$. Therefore, there is no root $\xi=i \omega$, with $\omega>0$ for (20), implying that the roots of (20) can not cross the purely imaginary axis. Thus all roots of (20) have a negative real parts provided $\mathrm{R}_{0}>1$. Then $\mathrm{E} 1$ is locally asymptotically stable. If $\mathrm{R}_{1}>1$, the eigenvalue $\xi_{2}$ is not negative, then $\mathrm{E}_{1}$ is unstable. 

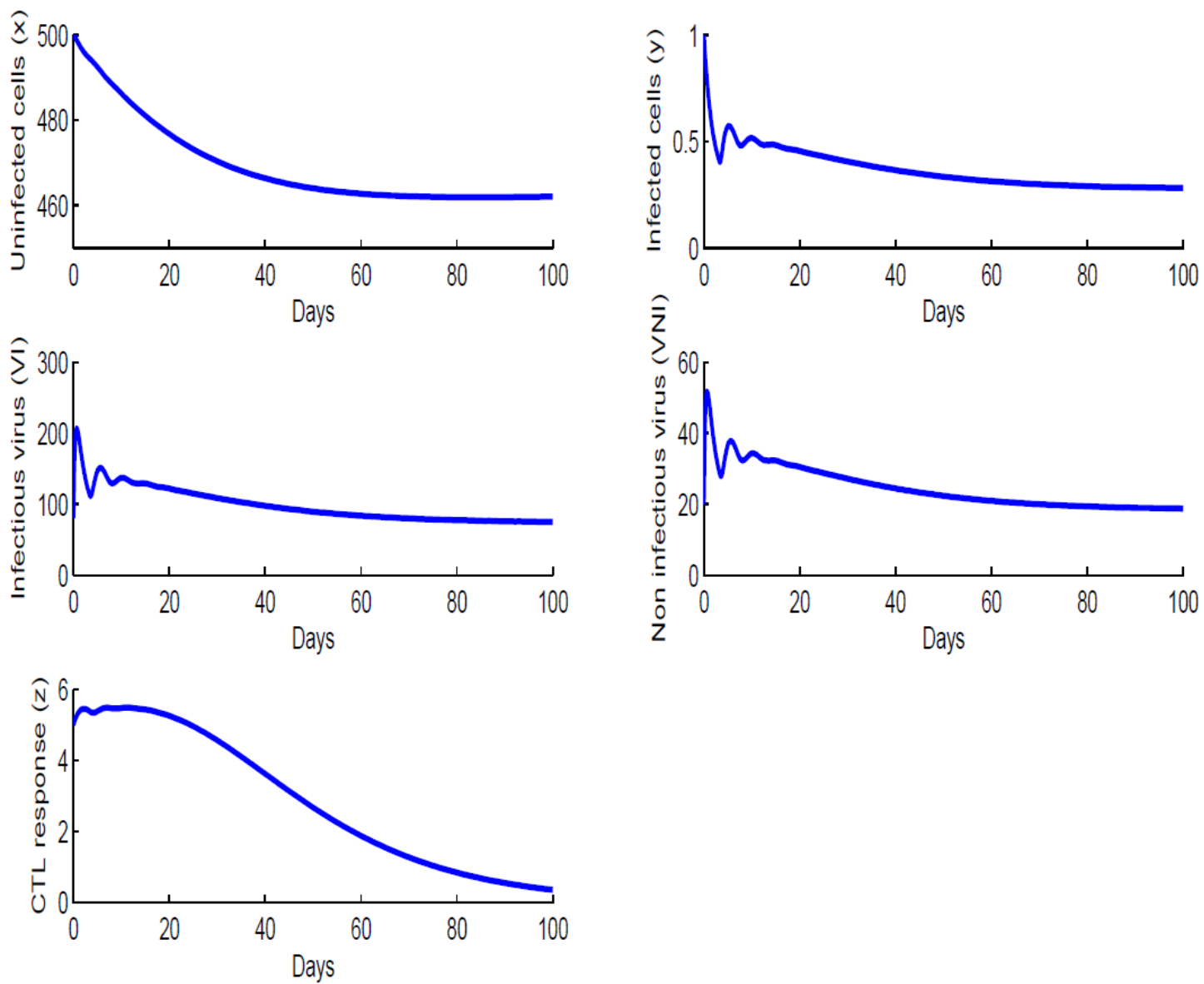

Figure 2: Shows the stability of $E 1$. The parameters are $\varepsilon=0.2, \eta=0.1, \lambda=10, \beta=0.000024$, $\mathrm{k}=1000, \mathrm{a}=0.5, \mathrm{r}=0.01, \mathrm{~d}=0.02, \mu=3, \mathrm{c}=0.2, \mathrm{~b}=0.1, \mathrm{p}=0.01 . \tau=3.3$ In this case, $\mathrm{R} 0=1.0845$, and $\mathrm{R} 1=0.9485$, and $\mathrm{E} 1(461.0347,0.2946,78.5524,19.6381,0)$

\subsection{Stability of the equilibrium with immune response}

Here we study the existence and stability of the endemic equilibrium point with immune response, E2. We can write E2 by

$$
\mathrm{E}_{2}=\left(\frac{\lambda}{d}\left(\frac{R_{1}}{R_{0}}\right), \frac{b}{c}, \frac{(1-\epsilon) k b}{\mu c}, \frac{\epsilon k b}{\mu c}, \frac{a+r}{p}\left(R_{1}-1\right)\right)
$$

It's clear that if $R_{1}<1$, the point $E_{2}$ does not exist and $E_{2}=E_{1}$ when $R_{1}=1$. We seek what will happen for the point $\mathrm{E}_{2}$ if $\mathrm{R}_{1}>1$.

From (17), the characteristic equation corresponding to E2 reduces to

$$
[\mu+\xi]\left[\xi^{4}++\tilde{a}_{1}(\tau) \xi^{3}+\tilde{a}_{2}(\tau) \xi^{2}+\tilde{a}_{3}(\tau) \xi+\tilde{a}_{4}(\tau)+\xi\left(\tilde{b}_{1}(\tau) \xi+\tilde{b}_{2}(\tau)\right) e^{-\xi \tau}\right]=0
$$

as the eigenvalue $\xi 1=-\mu$ of (24 ) is negative, then the stability of E2 is determined by the distribution of the roots of equation

$$
\left[\xi^{4}+\tilde{a}_{1}(\tau) \xi^{3}+\tilde{a}_{2}(\tau) \xi^{2}+\tilde{a}_{3}(\tau) \xi+\tilde{a}_{4}(\tau)+\xi\left(\tilde{b}_{1}(\tau) \xi+\tilde{b}_{2}(\tau)\right) e^{-\xi \tau}\right]=0
$$


where

$$
\begin{aligned}
& \tilde{a}_{1}(\tau)=\mu+\frac{d M}{c \lambda}+(a+r) R_{1} \\
& \tilde{a}_{2}(\tau)=\frac{d(a+r)\left(R_{1} M\right)}{c \lambda}+\mu\left[\frac{d M}{c \lambda}+(a+r) R_{1}\right]+b(a+r)\left(R_{1}-1\right) \\
& \tilde{a}_{3}(\tau)=\frac{d \mu(a+r)\left(R_{1} M\right)}{c \lambda}+b(a+r)\left(R_{1}-1\right)\left[\frac{d M}{c \lambda}+\mu\right] \\
& \tilde{a}_{4}(\tau)=\frac{d b \mu(a+r) M\left(R_{1}-1\right)}{c \lambda} \\
& \tilde{b}_{1}(\tau)=-\left((a+r) \mu R_{1}+\frac{d b r(a+r) R_{0}}{c \lambda}\right) \\
& \tilde{b}_{2}(\tau)=-\frac{(a+r) d \mu}{c \lambda}\left[b r R_{0}+c \lambda R_{1}\right]
\end{aligned}
$$

and $M=c \lambda+R_{0} b(a+r) e^{a \tau}$.

If $\tau=0$, equation (25) reduces to

$\mathrm{c \lambda} \quad[\mathrm{brR} 0+\mathrm{c \lambda R} 1]$

$$
\xi_{4}+\tilde{c}_{1} \xi_{3}+\tilde{c}_{2} \xi_{2}+\tilde{c}_{3} \xi+\tilde{c}_{4}=0
$$

where the coefficients $\tilde{c} \tilde{i}$ are obtained from the previous coefficients $a \tilde{i}(\tau)$ and bi $(\tau)$ by replacing $\tau$ by 0 .

We have already shown in [4], where there is no delay $(\tau=0)$, that the roots of equation (26) have negative real parts when $R_{1}>1$. Consequently $E_{2}$ is locally asymptotically stable when $R_{1}>1$, and $\tau=0$.

If $\tau>0$, the equation (25) has infinitely many roots. By Rouche's Theorem and continuity in $\tau$, the sign of the roots of equation (25) will change if it crosses imaginary axis, i.e. if Eqs (25) has purely imaginary root. Hence, we put $\xi=\mathrm{i} \psi$ a purely imaginary root of $(25)$, with $\psi>0$, and separate the real and imaginary parts, we have

$$
\begin{aligned}
\psi^{4}-\tilde{a}_{2} \psi^{2}+\tilde{a}_{4} & =-\tilde{b}_{1} \psi^{2} \cos (\tau \psi)-\tilde{b}_{2} \psi \sin (\tau \psi) \\
-\tilde{a}_{1} \psi^{3}+\tilde{a}_{3} \psi & =-\tilde{b}_{2} \psi \cos (\tau \psi)+\tilde{b}_{1} \psi^{2} \sin (\tau \psi)
\end{aligned}
$$

where the a $\tilde{i}$ and $\sim$ bi are the previous a $\mathrm{i}(\tau)$ and $\sim \mathrm{bi}(\tau)$.

Squaring and adding Eqs (27) and (28), we have

where

$$
\psi^{8}+\mathrm{e}_{1} \psi^{6}+\mathrm{e}_{2} \psi^{4}+\mathrm{e}_{3} \psi^{2}+\mathrm{e}_{4}=0
$$

$$
\begin{aligned}
& e_{1}=\tilde{a}_{1}^{2}-2 \tilde{a}_{2} \\
& e_{2}=\tilde{a}_{2}^{2}+2 \tilde{a}_{4}-2 \tilde{a}_{1} \tilde{a}_{3}-\tilde{b}_{1}^{2} \\
& e_{3}=\tilde{a}_{3}^{2}-2 \tilde{a}_{2} \tilde{a}_{4}-\tilde{b}_{2}^{2} \\
& e_{4}=\tilde{a}_{4}^{2}
\end{aligned}
$$

Let $Y=\psi^{2}$ then (29) becomes

$$
\mathrm{G}(\mathrm{Y})=\mathrm{Y}^{4}+\mathrm{e}_{1} \mathrm{Y}^{3}+\mathrm{e}_{2} \mathrm{Y}^{2}+\mathrm{e}_{3} \mathrm{Y}+\mathrm{e}_{4}=0
$$

The first and second derivative of function $\mathrm{G}$ are respectively. and $G^{\prime \prime}(Y)=12 Y^{2}+6 e_{1} Y+2 e_{2}$ he minimum value of $G^{\prime \prime}$ is $G^{\prime \prime}\left(\frac{-6 e_{1}}{24}\right), \cdot 3 e_{1} Y^{2}+2 e_{2} Y+e_{3}$

$$
\begin{aligned}
G^{\prime \prime}\left(\frac{-6 e_{1}}{24}\right) & =\frac{-3}{4} e_{1}^{2}+2 e_{2} \\
& =\frac{-3}{4} \tilde{a}_{1}^{4}-\tilde{a}_{2}^{2}-3 \tilde{a}_{1}^{2} \tilde{a}_{2}+4 \tilde{a}_{4}-4 \tilde{a}_{1} \tilde{a}_{3}-2 \tilde{b}_{1}^{2} \\
& =\frac{-3}{4} \tilde{a}_{1}^{4}-\tilde{a}_{2}^{2}-3 \tilde{a}_{1}^{2} \tilde{a}_{2}-4 \mu\left[\frac{d \mu(a+r)\left(R_{1} M\right)}{q \lambda}+b(a+r) \mu\left(R_{1}-1\right)\right] \\
& -4\left[\frac{d M}{c \lambda}+(a+r) R_{1}\right] \tilde{a}_{3}-2 \tilde{b}_{1}^{2} .
\end{aligned}
$$


Then, in case where $\mathrm{R}_{1}>1$, we have $G^{\prime \prime}\left(\frac{-6 e_{1}}{24}\right)<0$, because all a $\mathrm{i}>0$ for $\mathrm{i} \in\{1,2,3,4\}$. Hence, since $G^{\prime \prime}$ is a polynomial of degree 2 and $\lim _{Y \rightarrow+\infty} G^{\prime \prime}(Y)=+\infty$ and $\lim _{Y \rightarrow-\infty} G(Y)=+\infty$, the equation $\mathrm{G}^{\prime \prime}(\mathrm{Y})=0$ has exactly two real solutions, that are $\quad \alpha_{1}=\frac{-3 e_{1}-\sqrt{9 e_{1}^{2}-24 e_{2}}}{6}$

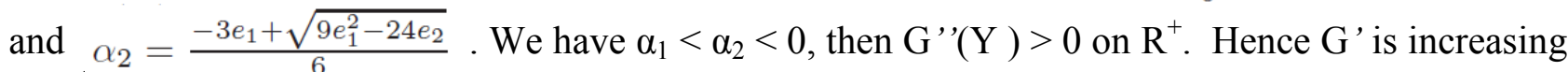
on $\mathrm{R}^{\prime}$. And $\mathrm{G}^{\prime}(0) \stackrel{6}{=} \mathrm{e}_{3}>0$ because

$$
\begin{aligned}
e_{3}=\left(b(a+r)\left(R_{1}-1\right)\left[\mu-\frac{d M}{c \lambda}\right]\right)^{2} & +2\left(d \mu^{2}(a+r)^{2}\right) \frac{d M}{c \lambda} R_{1}\left(R_{0}-R_{1}\right)+2(d \mu(a+r))^{2} R_{1}\left(R_{0}-R_{1}\right) \\
& +2 b(a+r) \mu\left(R_{1}-1\right) \frac{d M}{c \lambda}\left(\frac{1}{2}-\frac{d M}{c \lambda} \mu\right)^{2} \\
& +\frac{1}{2} b(a+r) \mu\left(R_{1}-1\right) \frac{d M}{c \lambda}
\end{aligned}
$$

and $\mathrm{R}_{0}>\mathrm{R}_{1}>1$.

Then $G(Y)>0$, therefore the function $G$ is increasing on $R^{+}$. Since $G(0)=e_{4}>0$, the equation (30) admits no solutions in $\mathrm{R}^{+}$. Thus, all roots of (25) have a negative real parts provided $\mathrm{R}_{1}>1$. Then, $\mathrm{E}_{2}$ is locally asymptotically stable. Thus, in the case where $\mathrm{R}_{1}>1$, we have the following theorem .

\section{Theorem 3}

If $R_{1}>1$, then $E_{2}$ is locally asymptotically stable for all $\tau>0$.
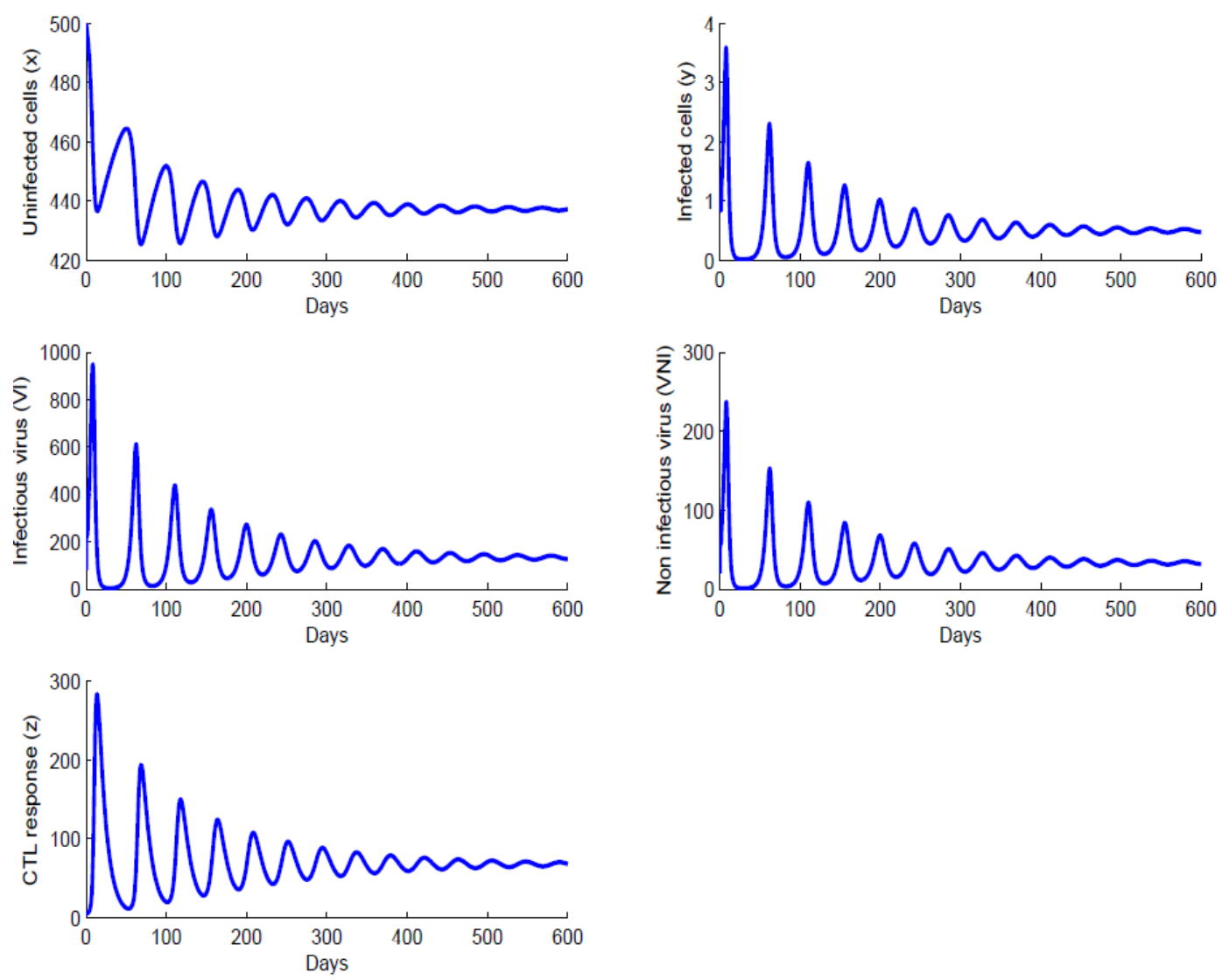

Figure 3: Shows the stability of E2. The parameters are $\varepsilon=0.1, \eta=0.2, \lambda=10, \beta=0.000024$, $\mathrm{k}=1000, \mathrm{a}=0.5, \mathrm{r}=0.01, \mathrm{~d}=0.02, \mu=3, \mathrm{c}=0.2, \mathrm{~b}=0.1, \mathrm{p}=0.01 . \tau=1.5 \mathrm{In}$ this case, $\mathrm{R} 0=2.6675$, and $\mathrm{R} 1=2.3329$, and $\mathrm{E} 2(437.2815,0.5,133.3333,33.3333,67.9769)$ 


\section{Discussion and Conclusion}

In this work, we gave a new mathematical model that describes HIV infection of CD4+ T-cells and immune response with intracellular delay and therapy with two drugs.

We conclude that to control the concentrations of the virus, a strategy should aim to reduce the value of R0 to below one. By the explicit formula for R0 $=\frac{(1-\theta) \lambda \beta k e^{-a \tau}}{(a+r) d \mu}$, we see that R0 can be decreased by increasing the efficacy of PIs and RTIs .

Also the intracellular delay plays a positive role in preventing the virus, because with all other parameters fixed, larger $\tau$ can bring $\mathrm{R} 0$ to a level lower than 1, making the infection free equilibrium point globally asymptotically stable (see figure (4)).

Figure (5) gives the following results:

$\mathrm{R}_{0}$ decreases in $\tau$ and $\mathrm{R}_{0}(\infty)=0$.

If $\tau \in\left[0,3.1942\right.$ [, then $\mathrm{R}_{0}>1$ and $\mathrm{R}_{1}>1$, and $\mathrm{E}_{2}$ exist and locally asymptotically stable.

If $\tau \in] 3.1942,3.4629$ [, then $\mathrm{R}_{0}>1$ and $\mathrm{R}_{1}<1$, and $\mathrm{E}_{1}$ exist and locally asymptotically stable.

If $\tau \in\left[3.4629,+\infty\left[\right.\right.$, then $\mathrm{R}_{0} \leq 1$, and $\mathrm{E}_{f}$ exist and globally asymptotically stable.
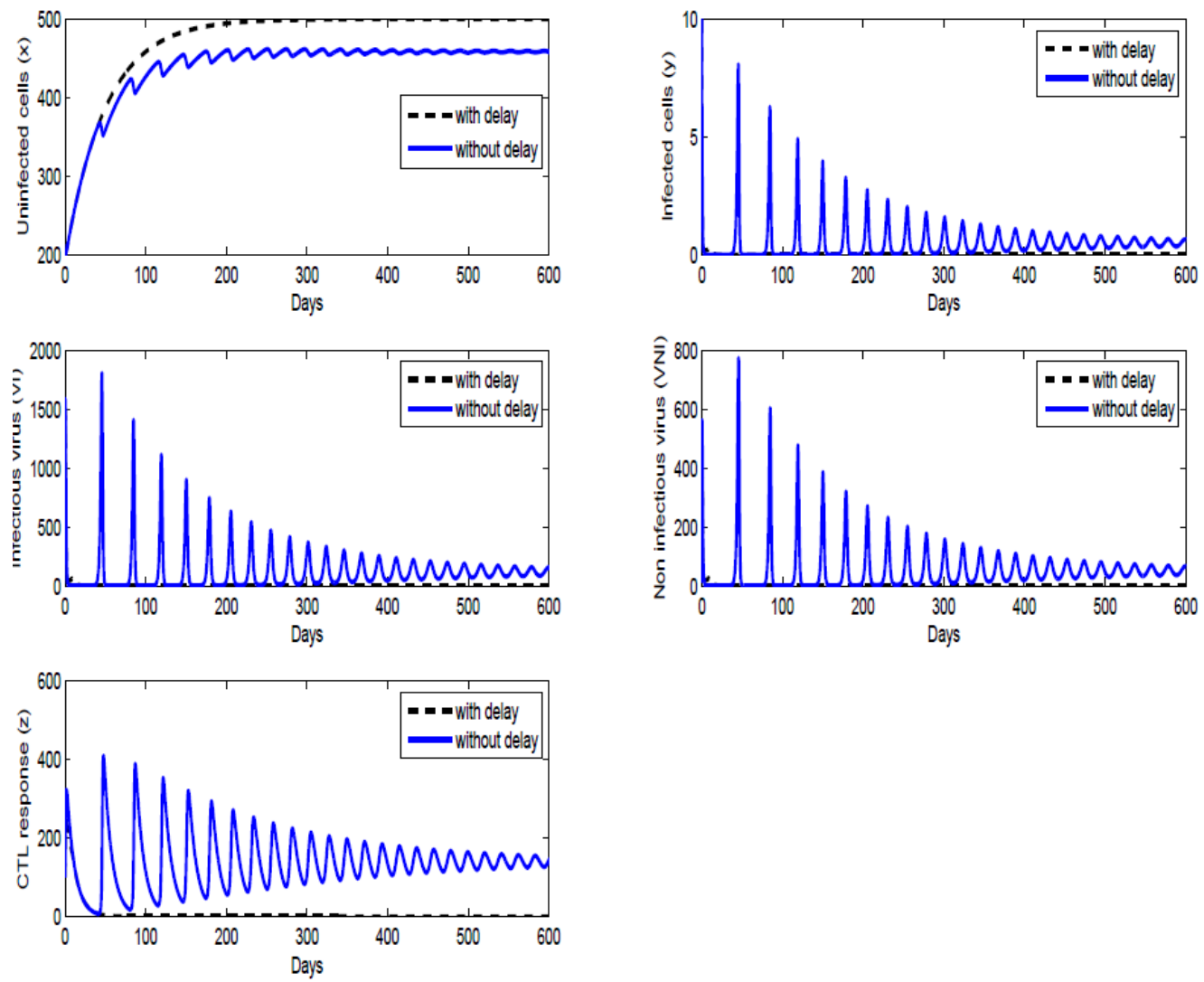

Figure 4: HIV population cell with and without intracellular delay. The parameters are $\varepsilon=0.3, \eta=0.35, \lambda=10, \beta=0.000024, \mathrm{k}=1000, \mathrm{a}=0.26, \mathrm{r}=0.01, \mathrm{~d}=0.02, \mu=3, \mathrm{c}=0.2$, $\mathrm{b}=0.1, \mathrm{p}=0.01$. In this case, the value of delay is $\tau=7.5$ 


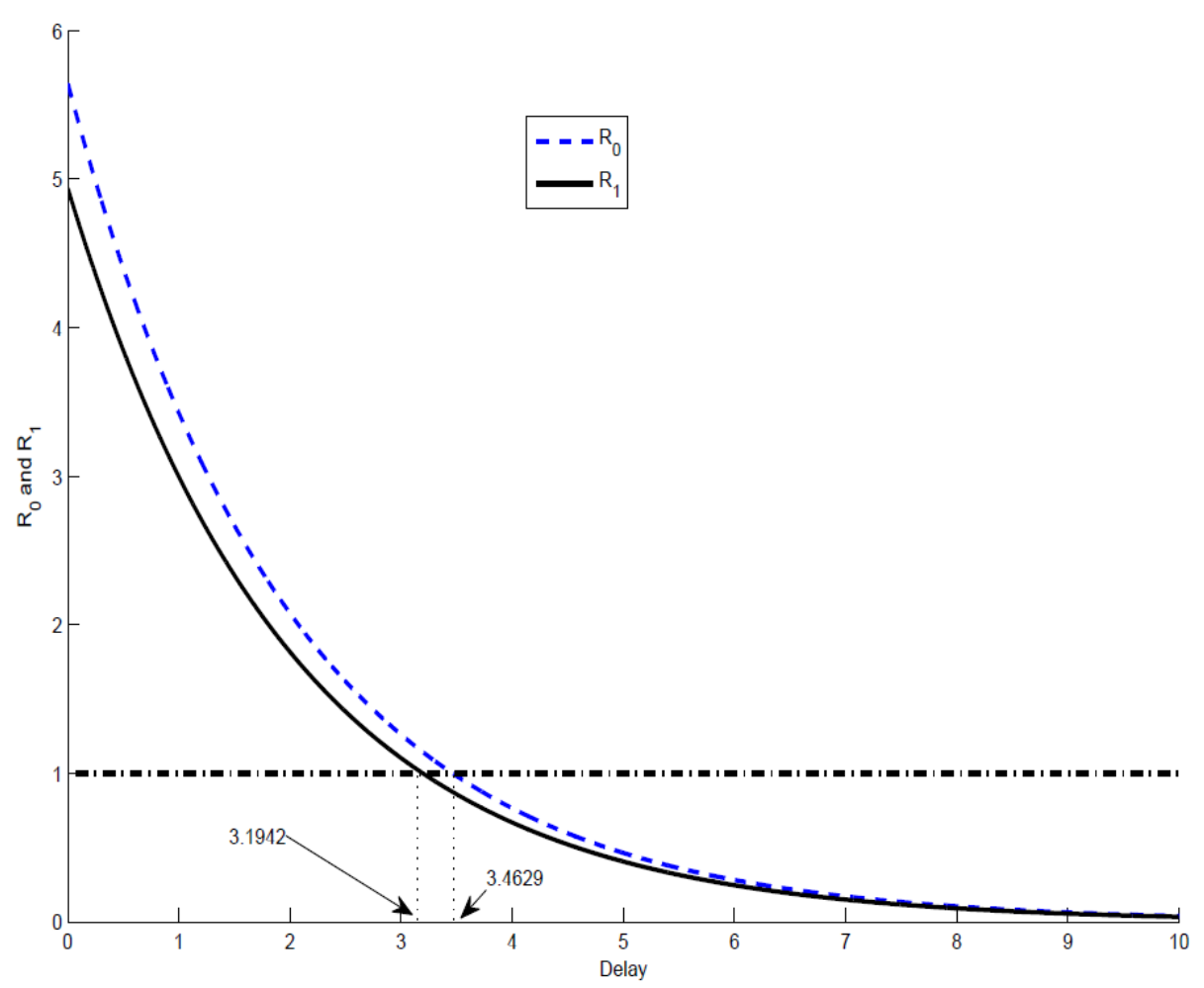

Figure 5: Gives R0 and R1 as a function of the delay $\tau$. The parameters are $\varepsilon=0.1, \eta=0.2$, $\lambda=10, \beta=0.000024, \mathrm{k}=1000, \mathrm{a}=0.5, \mathrm{r}=0.01, \mathrm{~d}=0.02, \mu=3, \mathrm{c}=0.2, \mathrm{~b}=0.1, \mathrm{p}=0.01$.

\section{References}

[1] S. Bonhoeffer, M. Rembiszewski, G. M. Ortiz and D. F. Nixon (2000). Risks and benefits of structured antiretroviral drug therapy interruptions in HIV-1 infection. AIDS , 14, 2313-2322.

[2] S. M. Ciupe, B. L. Bivort, D. Bortz, Nelson. P (2006), Estimates of kinetic parameters from HIV patient data during primary infection through the eyes of three different models, Math Biosci 200:1-27.

[3] R. V. Culshaw, S. Ruan,(2000). A delay-dierential equation model of HIV infection of CD4+T-cells. Math Biosci 165: 27-39.

[4] B. EL Boukari, K. Hattaf, N. Yousfi, (2013). Modeling the Therapy of HIV Infection with CTL Response and Cure Rate . Int. J. Ecol. Econ. Stat 28: 1-17.

[5] I. S. Gradshteyn, I. M. Ryzhik, (2000). Routh-Hurwitz Theorem, in Tables of Integrals, Series, and Products, 6th ed. San Diego, CA: Academic Press, 1076.

[6] J. Hale and S. M. Verduyn Lunel,(1993) Introduction to Functional Differential Equations, Springer- Verlag, New York .

[7] K. Hattaf and N. Yousfi, (2011). Dynamics of HIV Infection Model with Therapy and Cure Rate, International Journal of tomography and Statistics, 74-80.

[8] P. Nelson, A. Perelson, (2002). Mathematical analysis of delay differential equation models of HIV-1 infection, Mathematical Biosciences 179 73-94.

[9] M. A. Nowak, C. R. M. Bangham, (1996). Population dynamics of Immune Responses to Persitent Viruses, Science, 272, 74-79.

[10] A. S. Perelson, D. E. Kirschner, and R. D. Boer, (1993). Dynamics of HIV infection of CD4+ T-cells Math Biosci 114: 81-125. 
[11] A. Perelson, A. Neumann, M. Markowitz, J. Leonard and D. Ho, (1996). HIV-1 dynamics in vivo: Virion clearance rate, infected cell life-span, and viral generation time, Science, 271 , 1582-1586.

[12] A. Perelson, P. Nelson, (1999). Mathematical Analysis of HIV dynamics in vivo, SIAM Rev., 41 3-44.

[13] P. K. Srivastava, M. Banerjee, P. Chandra, (2010). A primary infection model for HIV and immune response with two discrete time delays, Diff. Equ. Dyn. Syst, 18(4): 385-399.

[14] L. Wang and M. Y. Li, Mathematical analysis of the global dynamics of a model for HIV infection of CD4+ T cells, Math. Biosci., 200 (2006), 44-57.

[15] N. Yousfi, K. Hattaf and A. Tridane, (2011). Modeling the adaptative immune response in HBV infection, J. Math. Bio., 63(5): 933-957

[16] H. Zhu and X. Zou, (2008). Impact of delays in cell infection and virus production on HIV1 dy- namics. Mathematical Medicine and Biology, 25, 99-112.

[17] H. Zhu and X. Zou, (2009). Dynamics of a HIV-1 Infection Model With Cell-Mediated Immune Response And Intracellular Delay, Discrete And Continuous Dynamical Systems Series B,Vol. 12, N o2, 511-524. 